\title{
Study of the Quality of Bee Product Tracking and Traceability System Based on Agent Technology
}

\author{
E. Yue*, Zhu Ye Ping, and Liu Sheng Ping \\ Research Institute of Agriculture Information Chinese Academy of Agricultural Science, \\ Beijing China 100081, \\ Tel.: +86-010-82109885; Fax: +86-010-82103120 \\ eyue@mail.caas.net.cn
}

\begin{abstract}
This paper is the use of modern information technology, research the quality of bee product tracking and traceability system based on Agent. On the one hand, through establish the quality of bee product tracking and traceability system, to provide a technical support and general-purpose tools, it includes bee products from production to sales for the entire process digital, visual expression, design, control and management. On the other hand, as a key technology research and exploration, the use of Agent technology, proposed intelligence and cooperative control methods about the quality and safety of bee products, and for the control of the quality and safety of bee products to provide new methods and ideas.
\end{abstract}

Keywords: Bee Product, Agent, Cooperative, Tracking, Traceability.

\section{Introduction}

Food security is the most concerned about people, it is the most direct and practical interests for people. It is research in recent years one of the hot issues how to effective control and management food safety. China is the largest country about bee-keeping, production and export in the world's, but it is not apiculture power, the main reason is: beekeeping information relative occlusion, information asymmetry about bee production, processing, transportation, storage, marketing and so on, the quality of bee products difficult to control. On the other hand, the role of human factors greatly lead to loosely organized, information management methods stay in the database management and query stage. Therefore, it is necessary use of the modern methods of information management and technology in the bee produce, it can build a new information and intelligent management model about beekeeping in China, so that the level of quality and safety of bee products can assured and enhanced in China through effective tracking and traceability.

This paper is the use of modern information technology, research the quality of bee product tracking and traceability system based on Agent. As a key technology research and exploration, the use of Agent technology, proposed intelligence and

\footnotetext{
${ }^{*}$ Corresponding author.
} 
cooperative control methods about the quality and safety of bee products, and for the control of the quality and safety of bee products to provide new methods and ideas.

From an engineering perspective, the bee product quality tracking and traceability system is a wide-area Internet-based technology and distributed artificial intelligence system. Agent technology can be used to solve the distributed system, it has many good features based on Agent and Multi-Agent technology application system, such as initiative, intelligence, interaction, collaboration and mobility and so on. In this paper is based on the Agent ideas and for the quality of bee product tracking and traceability system to explore.

\section{Agent Summarize}

Agent comes from distributed artificial intelligence (DAI) in 70s of the 20th century, and in other areas with a combination of various technologies, to expand from the DAI in its research field. It is generally believed that Agent should have the knowledge, goals and abilities. Agent knowledge is a described about its environment and the requirements of a solution of the problem by the user, it can obtain through the other Agent (in multi-Agent system) or their own learning. Agent goals is solution the problem of ways and means. Agent goals is can be defined as the form of multigroup: Agent $=(\mathrm{M}, \mathrm{K}, \mathrm{A}, \mathrm{I}, \mathrm{L}, \mathrm{S}, \mathrm{G}, \mathrm{F}, \mathrm{C})$, in this: $\mathrm{M}=$ method, $\mathrm{K}=$ knowledge, $\mathrm{A}=$ property, $\mathrm{I}=$ reasoning mechanism, L=language, $\mathrm{S}=$ transmission of information, $\mathrm{G}=$ overall knowledge, $\mathrm{F}=$ inheritance mechanism, $\mathrm{C}=$ system services, this is a detailed description of Agent(Yao Li, Zhang Wei Ming,2002). With the increase distribution and complexity of system, the individual Agent often can not independently achieve a number of complex systems due to their own knowledge, computing resource constraints, Therefore, multi-Agent technology is the rapid development become a new focus about DAI research field. Multi-Agent System (MAS) consists of a number of Agent, the use of parallel distributed processing technology and modular design, the complex system is divided into relatively independent subsystems, through the Agent of cooperation and competition accomplish the complex issues to solution.

\section{Bee Product Quality Tracking and Traceability System Architecture Based on Agent}

\subsection{System Architecture}

Bee Products (such as: honey, Royal Jelly, etc.) from field to home, it have a number of links includes the acquisition of bee products, bee product processing, transport and sale, each link exists between each other the exchange of information. Due to geographical differences (the management are widely distributed In different regions), the time difference (there is a huge time difference about access to products, access to relevant information), making information management has become very complicated. On the other hand, for the bee products own characteristics, such as pests and diseases, medication, heavy metal pollution have a serious impact on the quality factors, these factors must be the focus of inspection about bee product. 


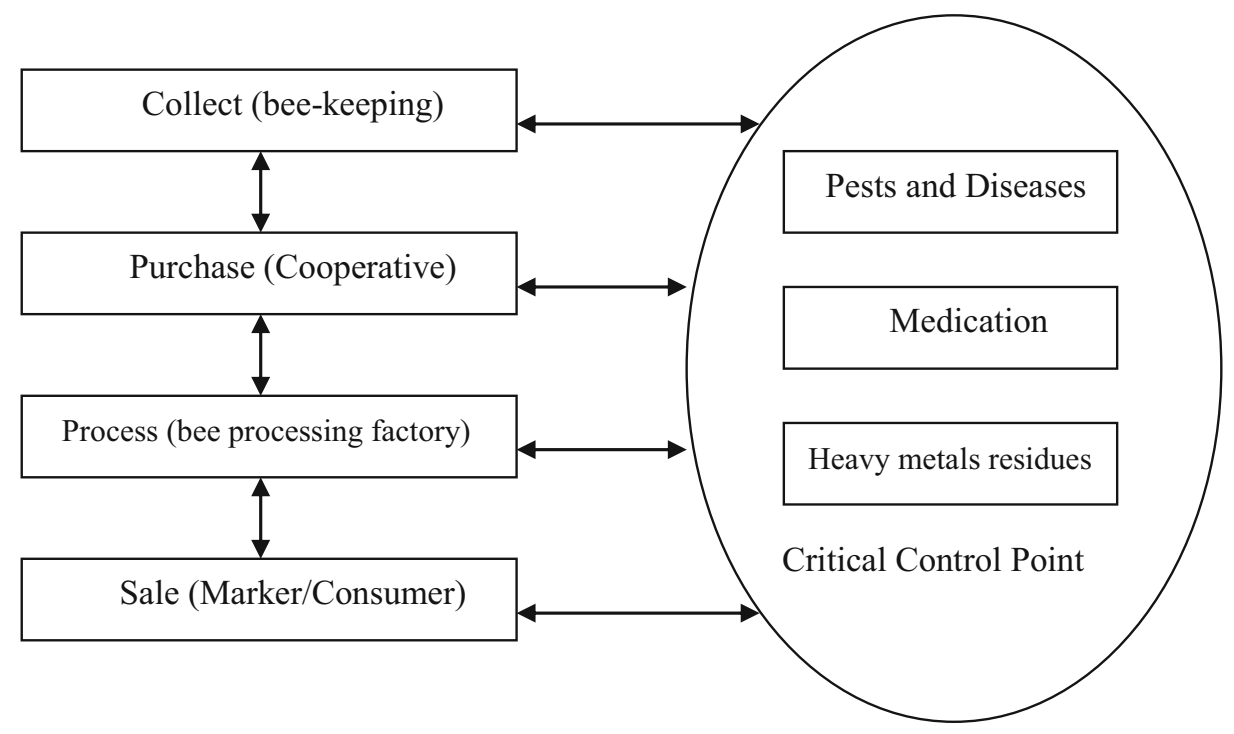

Fig. 1. Bee product quality tracking and traceability system architecture

Therefore, the quality of bee products tracking and traceability system can be decomposed as a dynamic system, no matter how large the scale, in theory, the system can be broken down into a number of interrelated sub-systems (such as acquisition system, processing systems, transport systems, marketing systems, etc.). There are also relatively independent subsystems in quality management in the actual work, the bee for the production, acquisition, processing, transportation and sales departments have the corresponding entities. On the other hand, As a result of its own characteristics, Critical Control Point(such as pests and diseases, medication, heavy metals) play a very important role in the quality of bee products tracking and traceability system, the advantages and disadvantages of these entities directly related to the quality of bee products. These entities can completed their own tasks and goals to achieve optimal, they have independent thinking and ability to fulfill its mandate, they can cognitive state that all other entities of the current, and can accept the request and orders of other entities to change their behavior mechanism.

Based on the above analysis, the author proposed a bee product quality tracking and traceability system modeling framework architecture. The entire model is built based on TCP/IP protocol on the conceptual level, it is divided into application-layer, Agent layer, network layer platforms.

(1) Application Layer: It responsible for user interaction. Its functions include two aspects: (1) Accept the request of the user, (2) Submit the query results in accordance with the requirements of users. Application layer will be the user's request in accordance with the Agent layer organize, and then put it to the Agent layer.

(2) Agent layer: it is the most complex part of the core in the whole system. For the Application Layer, in accordance with the requirements of users to generate user's 


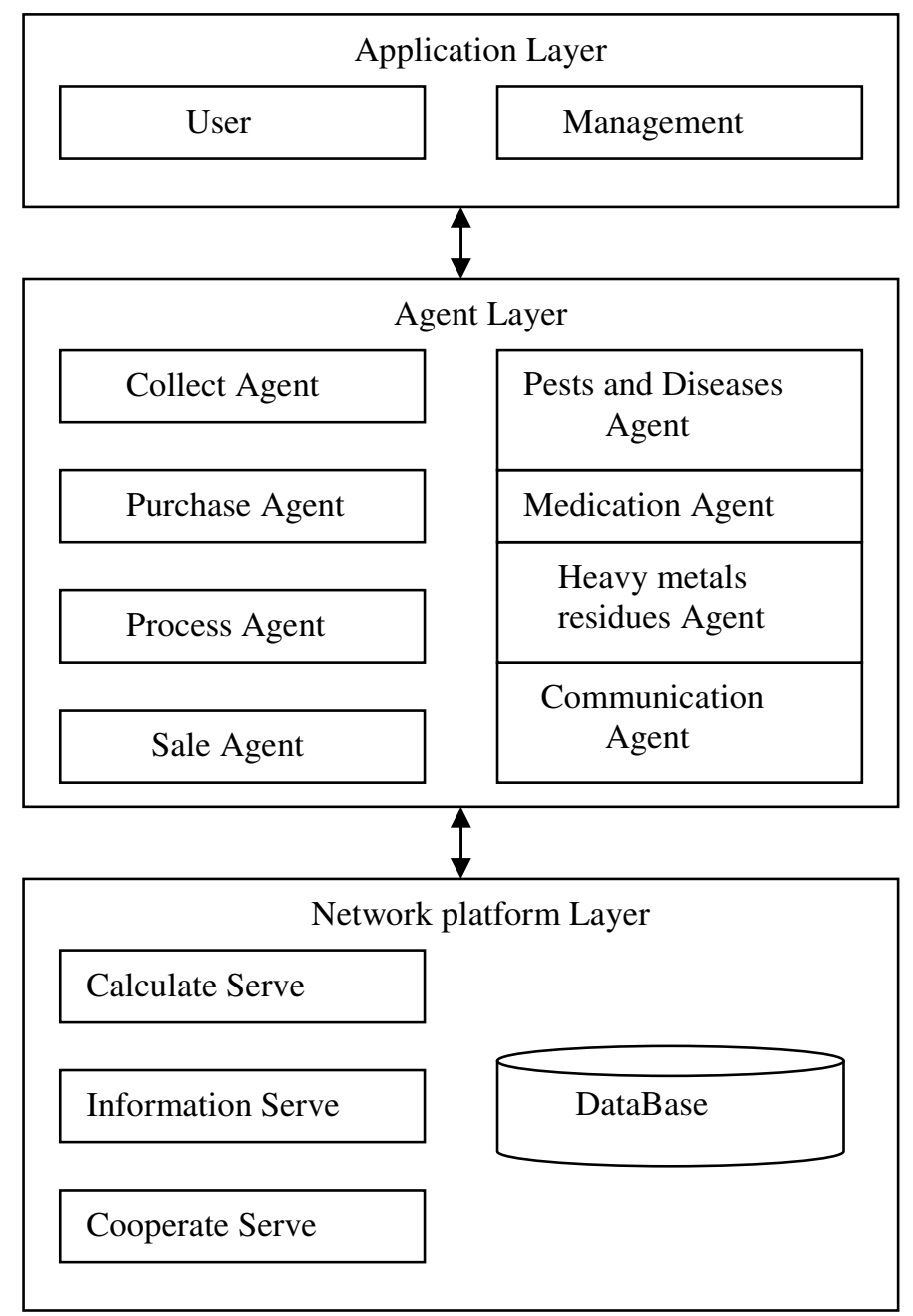

Fig. 2. System architecture based on Agent

Intelligent Agent object, At the same time, it also responsible for the management of all issued from the local Agent Object, and record their current location and status, and completed the collection of functions and returned to the application layer. For the network platforms layer, it transmission the Agent Object to the platform.

(3) Network layer platforms: It is the whole system of transmission pipelines, it provides the environment for the survival Agent, it is the basis for Agent Mobile, is a place of communication between the Agent. Platform network layer is based on the existing network communication protocols, it transmit data and receive data streams through consultations Port. 


\subsection{Multi-Agent Communication}

Knowledge query and manipulation language (KQML) was widely used as a communication language of the Agent, it is becoming the standard for communication of Agent. KQML is a descriptive language about exchange knowledge and information, It defines the mode of impart information and Message handling agreement in Agent, By providing a standard of communication language to achieve the exchange of information and knowledge sharing between the Agent (George F Luger, 2004).

Agent communication model and multi-Agent collaboration is closely related to the organization. In essence, all the multi-Agent systems are distributed architecture, each of Agent are cooperation and collaboration, there is no relationship between the subordinate and control. Each of Agent have their own independent goals, aspirations and behavior, it can choose partners, and accept or reject the task. In this case, most of the problems about multi-Agent cooperative must be members of Agent own knowledge, reasoning, social, adaptation, learning ability to solve, which makes the design of Agent complicated entities (Wang Long, Zhang Yi, 2003).

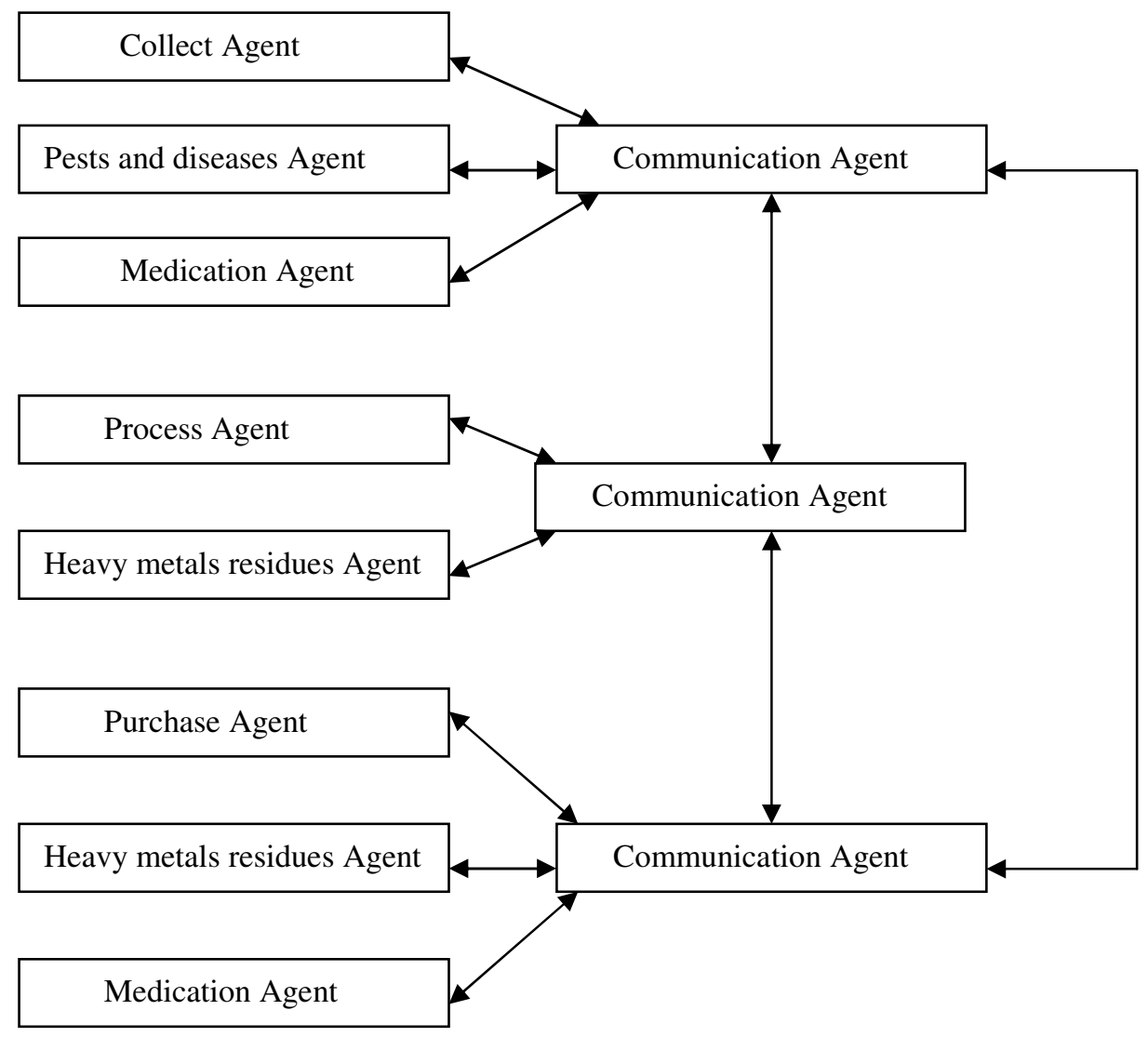

Fig. 3. Multi-Agent Communication Architecture 
Bee Products Quality Tracking and traceability information system is a complex multi-Agent system, the organization of ideas from the Internet, as shown in Figure 4 using the communication mode.

First of all, the Agent classification, will be in the same geographic or Agent of the same functions of a domain Agent group, each Agent group establish a communications agent (Collaborative Communications Agent).

Second, Agent of the Agent group only communication with the communications agent (Collaborative Communications Agent) and find the object of communication.

Third, when there is a suitable Agent Object on the same Agent group, Agent by sending and receiving KQML messages to communicate with other Agent.

Fourth, When there is a suitable Agent object in other Agent groups, the communication process must join the other group of Agent communication agent(Collaborative Communications Agent). Communications agents do not know or explain the content of KQML messages, this improve the system's security and scalability.

The distribution and concentration of mixture communication construct a Union Agent group, which not only resolved the Agent to manage the complexity of communication, but also resolved the Agent time-consuming problem when the large of communication.

\section{Discussion}

Bee Products Quality tracking and traceability System is a complex systems about supply chain for all members of the management, the purpose is monitor every aspect of product quality in the supply chain, at the same time achieve maximum customer satisfaction. It faces the environment including unpredictable and rapidly changing market demand, product mix and production re-configurations, a variety of different types of computer hardware and software systems and different business rules and the production of technical specifications. In the Bee product quality tracking and traceability process, due to its software and hardware infrastructure and its users is distribution, dynamics and uncertainty, so it is very difficult to adapt to the needs of the management integration, The use of software agent technology in the tactical level and operational level to carry out an effective management will become the direction of the research. The main method is to use multi-agent system architecture, through consultations between the agent, to complete all the tasks together. At the same time, the mobile agent is a new research direction, it is being applied to solve the distributed and heterogeneous environment of collaboration and management. Mobile agent not only has the autonomy, communications and reflect, and it can move freely in the network, it can be brought about by their own information "push" out, but also will require the collection and processing of information " pull "come in, so it is possible to making the information in the supply chain into the rapid flow. In this paper, the application of mobile agent technology in the supply chain to achieve the quality tracking and traceability bee management, aimed at taking advantage of its own characteristics (autonomy, intelligence, initiative and mobility), allows information to flows more effectively in Agent group. 


\section{Conclusion}

Based on the analysis of quality control of bee products, combined with bee management system characteristics, the introduction of agent technology, Put forward the theme of bee products quality management system model based on Agent, and through the introduction of agent technology to achieve the feasibility of the model. Described the system model of the structure and function modules in this paper. Through functions research of the Agent and analysis of communications patterns, it can be seen: bee product quality tracking and traceability information system based on Agent research and development have the prospect of important applications and practical value.

\section{Acknowledgements}

This research was supported by National Scientific and Technical Supporting Programs Funded by Ministry of Science and Technology of China (nyhyzx07-041).

\section{References}

Li, Y., Ming, Z.W.: Intelligent and Cooperative Information Systems. Publishing of Electronics Industry, Beijing (2002)

Luger, G.F.: Artificial intelligence. Publishing of Electronics Industry, Beijing (2004)

Long, W., Yi, Z.: Key Techniques to Realize Cooperation of Mobile Agent in MAS Environment. School of Computer Science and Engineering 32(2), 158-163 (2003)

Trappey, A.J.C., Trappey, C.V., Hou, J.: Mobile agent technology and application for online global logistic services. Industrial Management \& Data System 104(1/2), 169-183 (2004)

Ming, Z.J., Peng, Z.X.: An agent-oriented requirement analysis and modeling method 4, 33-35 (November 2006)

Jing, Z.: Bee product quality and safety analysis of the key technology research and development. China apiculture 57(12), 30-32 (2006)

DeLoach, S.A.: Engineering Organization-based Multiagent Systems. In: Garcia, A., Choren, R., Lucena, C., Giorgini, P., Holvoet, T., Romanovsky, A. (eds.) SELMAS 2005. LNCS, vol. 3914, pp. 109-125. Springer, Heidelberg (2006)

Chang, M.-H., Harrington Jr., J.E.: Agent-based models of organizations. In: Handbook of Computational Economics II (2006)

Yi, S.C.: Computing based on Agent. Publishing of Tsinghua University, Beijing (2007)

Zhi, S.Z.: Advanced Artificial Intelligence. Publishing of Science (2006) 